\title{
Nicotinamide mononucleotide increases muscle insulin sensitivity in prediabetic women
}

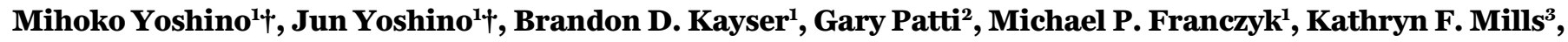 \\ Miriam Sindelar ${ }^{2}$, Terri Pietka', Bruce W. Patterson', Shin-Ichiro Imai ${ }^{3}$, Samuel Klein ${ }^{1 *}$
}

${ }^{1}$ Center for Human Nutrition, Washington University School of Medicine, St. Louis, MO, USA. ²Department of Chemistry, Washington University School of Medicine, St. Louis, MO, USA. ${ }^{3}$ Department of Developmental Biology, Washington University School of Medicine, St. Louis, MO, USA.

†These authors contributed equally to this work. *Corresponding author. Email: sklein@wustl.edu

In rodents, obesity and aging impair nicotinamide adenine dinucleotide (NAD+) biosynthesis, which contributes to metabolic dysfunction. Nicotinamide mononucleotide (NMN) availability is a rate-limiting factor in mammalian NAD+ biosynthesis. We conducted a 10-week, randomized, placebo-controlled, doubleblind trial to evaluate the effect of NMN supplementation on metabolic function in postmenopausal women with prediabetes who were overweight or obese. Insulin-stimulated glucose disposal, assessed by using the hyperinsulinemic-euglycemic clamp, and skeletal muscle insulin signaling (phosphorylation of AKT and mTOR) increased after NMN supplementation, but did not change after placebo treatment. NMN supplementation up-regulated the expression of platelet-derived growth factor receptor $\beta$ and other genes related to muscle remodeling. These results demonstrate NMN increases muscle insulin sensitivity, insulin signaling and remodeling in women with prediabetes who are overweight or obese (ClinicalTrial.gov NCT 03151239).

Nicotinamide adenine dinucleotide $\left(\mathrm{NAD}^{+}\right)$is a co-substrate for $\mathrm{NAD}^{+}$-consuming enzymes that are essential in the regulation of diverse biological processes. In rodents, inadequate $\mathrm{NAD}^{+}$biosynthesis in liver, adipose tissue and skeletal muscle contributes to the pathogenesis of obesity- and aging-associated metabolic abnormalities, including insulin resistance, $\beta$-cell dysfunction, and hepatic steatosis (1-9). The primary pathway for $\mathrm{NAD}^{+}$biosynthesis involves the conversion of nicotinamide to nicotinamide mononucleotide (NMN) and subsequent conversion of $\mathrm{NMN}$ to $\mathrm{NAD}^{+}(5)$. The production of NMN is the key rate-limiting factor in mammalian $\mathrm{NAD}^{+}$ biosynthesis. Systemic NMN administration in obese mice fed a high-fat diet increases tissue $\mathrm{NAD}^{+}$concentrations and improves glucose tolerance, insulin sensitivity, and $\beta$-cell function $(1,4,10)$, and long-term NMN administration in mice fed regular chow mitigates age-associated insulin resistance (11). The beneficial effects of NMN supplementation in rodents has led to rapid commercial development of NMN products for people, and NMN is marketed in the United States and other countries as a supplement that improves glucose control, enhances energy metabolism and reverses the metabolic complications of aging. Although NMN is present in natural foods, including edamame, broccoli, avocado, tomatoes, and milk, the daily amount of NMN that is normally consumed as part of a healthy diet is likely less than 2 $\mathrm{mg} /$ day (5). The dose of NMN available in commercial products ranges from $50 \mathrm{mg}$ to $150 \mathrm{mg} /$ capsule, and some consumers take two $150 \mathrm{mg}$ capsules daily. However, we are not aware of studies on the metabolic effects of daily NMN supplementation in people.

We conducted a 10-week, randomized, placebo-controlled, double-blind trial in postmenopausal women with prediabetes who were overweight or obese (body mass index 25.3 to $39.1 \mathrm{~kg} / \mathrm{m}^{2}$ ) to determine the effects of NMN supplementation on: i) body composition, ii) skeletal muscle insulin sensitivity, and insulin signaling; and iii) muscle $\mathrm{NAD}^{+}$content and global gene expression profile. We specifically studied postmenopausal women because NMN treatment caused greater beneficial metabolic effects in female than male mice with diet-induced diabetes (1).

Twenty-five postmenopausal women with prediabetes based on criteria proposed by the American Diabetes Association (12) who were overweight or obese completed this study; 12 were randomized to the placebo group and 13 to the NMN group (250 mg/day; Oriental Yeast Co., Ltd., Tokyo, Japan) (fig. S1). Baseline testing included: i) dual-energy x-ray absorptiometry and magnetic resonance imaging to assess body composition; ii) a hyperinsulinemic-euglycemic clamp procedure (4-hour basal stage and 4-hour insulin infusion at $50 \mathrm{mU} / \mathrm{m}^{2} / \mathrm{min}$ ), in conjunction with stable isotope glucose and palmitate tracer infusions to assess multi-organ insulin sensitivity; and iii) percutaneous biopsies of quadriceps muscle during the basal period and insulin infusion to assess cellular effects of NMN treatment. After baseline studies were completed, participants were randomly assigned to 10 weeks of treatment with placebo or NMN. After participants 
completed $\sim 10$ weeks of treatment, all study procedures conducted at baseline were repeated. In addition, peripheral blood mononuclear cell (PBMC) $\mathrm{NAD}^{+}$content was assessed for 4 hours after placebo or NMN ingestion. Based on weekly pill counts, $99.6 \%$ of prescribed pills in the placebo group and $100 \%$ of prescribed pills in the NMN group were taken by the participants. No adverse events were reported and no abnormalities in standard blood tests were detected in either group (table S1).

Plasma concentrations of N-methyl-2-pyridone-5-carboxamide and N-methyl-4-pyridone-5-carboxamide, which are metabolites produced by NMN (13), increased after 10 weeks of NMN treatment, but not placebo (Fig. 1A). Basal NAD ${ }^{+}$content in PBMCs increased after 10 weeks of NMN treatment, but did not change after placebo treatment (Fig. 1B). Ingestion of a single $250 \mathrm{mg}$ dose of NMN at the end of 10 weeks of treatment did not cause a further increase above basal in $\mathrm{PBMC} \mathrm{NAD}^{+}$content, assessed in serial blood samples obtained for 240 min after ingestion (Fig. 1C); however, the 240min $\mathrm{PBMC} \mathrm{NAD}^{+}$content area-under-the-curve above zero was $43 \%$ greater $(\mathrm{p}<0.01)$ in the NMN group than in the placebo group because of the higher basal value in the NMN group (Fig. 1D). Quadriceps muscle tissue samples were obtained 1.5 hours after the last dose of placebo or NMN to assess the effect of treatment on skeletal muscle NMN metabolites. Although muscle $\mathrm{NAD}^{+}$and nicotinamide content did not change after 10 weeks of treatment in either group (Fig. 1, E and F), muscle N-methyl-nicotinamide, methyl-2-pyridone-5-carboxamide, and N-methyl-4-pyridone-5carboxamide increased after NMN but not placebo treatment (Fig. 1, G to I), suggesting NMN treatment increased muscle $\mathrm{NAD}^{+}$turnover.

Body composition (fat mass, fat-free mass, intra-abdominal adipose tissue volume, and intrahepatic triglyceride content), blood pressure, plasma glucose, insulin, free fatty acid, lipids, adiponectin and leptin concentrations, and both basal glucose and fatty acid kinetics did not change in either group after 10 weeks of treatment (Table 1). Muscle insulin sensitivity, assessed as the rate of insulin-stimulated glucose disposal per $\mathrm{kg}$ of fat-free mass during the clamp procedure, was $25 \pm 7 \%$ greater after than before 10 weeks of NMN supplementation $(\mathrm{p}<0.01)$, but was not different after than before placebo treatment (Fig. 2A). In contrast, hepatic insulin sensitivity [assessed as the hepatic insulin sensitivity index (14) and the suppression of glucose rate of appearance (Ra) during insulin infusion] and adipose tissue insulin sensitivity [assessed as the adipose tissue insulin resistance index (15) and the suppression of palmitate Ra] were not different after than before treatment with either placebo or NMN (fig. S2).

We evaluated the effect of NMN supplementation on phosphorylation of serine 473 (Ser473) and threonine 308 (Thr308) of the protein kinase AKT and phosphorylation of
Ser2448 of mTOR in muscle, which are key components of the insulin signaling pathway involved in regulating glucose uptake and muscle remodeling. Muscle AKT and mTOR phosphorylation and total AKT and mTOR protein abundance during insulin infusion were greater after than before treatment in the NMN group, but did not change in the placebo group (Fig. 2, B and C, and fig. S3). These cellular findings are consistent with the effects of placebo and NMN treatment on muscle insulin sensitivity observed in vivo.

We used RNA-sequencing to evaluate global gene expression of quadriceps muscle samples. We identified differentially-expressed genes (DEGs, False Discovery Rate <0.05) after versus before 10 weeks of placebo or NMN treatment during basal conditions and insulin infusion (Fig. 3A and table S2). During the basal conditions, there were 21 DEGs in the placebo group and 15 DEGs in the NMN group after compared with before treatment (Fig. 3A and table S2). During insulin infusion, there were 5 DEGs in the placebo group and 308 DEGs in the NMN group after compared with before treatment; none of the DEGs in the NMN group was affected by placebo treatment (Fig. 3A and table S2).

Database for Annotation, Visualization and Integrated Discovery pathway analysis was used to identify the gene ontology (GO) terms significantly enriched (adjusted P-value $<0.05$ ) with the 308 DEGs during insulin infusion after NMN treatment. The "Platelet-derived growth factor (PDGF) binding" pathway was the most highly enriched (Fig. 3B and table S3). DEGs were significantly enriched in biological pathways of collagen and extracellular matrix metabolism, which are downstream elements of PDGF signaling (Fig. 3B and table S3). Moreover, NMN supplementation significantly up-regulated skeletal muscle expression of PDGF receptor $\beta$ $(P D G F R \beta)$ and markers of skeletal muscle pericytes (CD9O and CD109) during insulin infusion (Fig. 3C). Supplementation with NMN also increased the expression of other genes related to myogenic PW1-positive interstitial cells and pericytes (PW1/PEG3, QPRT, NG2, and PECAM1) (16, 17), but these increases did not achieve statistical significance (fig. S4). In addition, downstream targets of PDGF signaling, such as COL1A1, COL5A1, and COL6A1, were significantly up-regulated after NMN treatment (Fig. 3C). These results indicate that NMN treatment increased myogenic cell populations and enhanced PDGF signaling in skeletal muscle.

Because of the importance of $\mathrm{NAD}^{+}$biology on mitochondrial function and the relationship between muscle mitochondria and muscle function $(3,18)$, we evaluated whether NMN affected skeletal muscle mitochondrial respiratory capacity and physical function. Muscle mitochondrial oxidative capacity, assessed by using high-resolution respirometry of quadriceps muscle samples obtained by percutaneous biopsy, did not change after 10 weeks of treatment with either placebo or NMN (fig. S5). Muscle physical function, assessed by 
measuring handgrip strength and torque, fatigability and recovery from fatiguing exercise of knee extensors and flexors of the dominant leg, were not affected by 10 weeks of placebo or NMN treatment (fig. S6).

This randomized, placebo-controlled, double-blind trial demonstrates that 10 weeks of NMN supplementation increases muscle insulin signaling (increased insulin-stimulated phosphorylated AKT and mTOR) and muscle insulin sensitivity (increased insulin-stimulated glucose disposal rate expressed per $\mathrm{kg}$ fat-free mass) in postmenopausal women with prediabetes who are overweight or obese. This improvement in muscle insulin sensitivity is clinically relevant and is similar to the improvement observed after $\sim 10 \%$ weight loss (19) and after 12 weeks of treatment with the insulin sensitizing agent troglitazone (20) in people with obesity. The increases in muscle insulin-stimulated AKT phosphorylation and glucose uptake after NMN treatment observed in our participants are consistent with studies conducted in rodent models $(1,3,11)$. Our data suggest an NMN-induced increase in muscle $P D G F R \beta$ expression could be involved in mediating this effect, because PDGF signaling, particularly through PDGFR $\beta$, enhances insulin-stimulated AKT phosphorylation and glucose transport in skeletal muscle and multiple cell types (21-23). The absence of changes in steady-state levels of muscle $\mathrm{NAD}^{+}$in our NMN-treated participants is also consistent with studies conducted in mice that found NMN administration improved insulin sensitivity without changing muscle $\mathrm{NAD}^{+}$concentration (11). However, NMN treatment increased the levels of muscle NMN metabolites (N-methyl nicotinamide, N-methyl-2-pyridone-5-carboxamide, and $\mathrm{N}$ methyl-4-pyridone-5-carboxamide), suggesting NMN treatment increased skeletal muscle $\mathrm{NAD}^{+}$turnover. The effect of NMN was specific to insulin sensitivity in muscle and did not affect other important variables associated with insulin resistance, including indices of liver and adipose tissue insulin sensitivity, intra-abdominal adipose tissue volume, intrahepatic triglyceride content, and fasting plasma glucose, insulin and adiponectin concentrations. These results demonstrate that NMN has selective beneficial effects on insulin-mediated glucose metabolism in skeletal muscle.

Our data demonstrate a robust effect of NMN on skeletal muscle biology. The data obtained from our unbiased global transcriptome profiling of skeletal muscle demonstrate that, compared with placebo, NMN caused a 60-fold increase in the number of DEGs during insulin infusion. The PDGF binding pathway was the most highly enriched with DEGs, and muscle PDGFR $\beta$ and downstream targets of PDGF signaling were significantly up-regulated during insulin infusion after NMN treatment. In addition, NMN treatment increased insulin-stimulated mTOR phosphorylation and gene expression of selected markers of myogenic PW1/PEG3-positive interstitial cells and pericytes $(16,17)$, which together indicate increased skeletal muscle remodeling and regeneration.

In contrast to the insulin-sensitizing effects of NMN we observed in our participants, the results from several randomized controlled trials conducted in middle-age and olderadult men found that treatment with nicotinamide riboside $(\mathrm{NR})$, another $\mathrm{NAD}^{+}$intermediate that improves insulin sensitivity in rodents $(5,8,24)$, did not affect whole-body or muscle insulin sensitivity (25-27). The reason for the absence of metabolic benefits of NR supplementation in these clinical studies is not known, and further studies are needed to address this issue.

The results from our study demonstrate NMN supplementation $(250 \mathrm{mg} /$ day) increases skeletal muscle insulin signaling, insulin sensitivity, and muscle remodeling in postmenopausal women with prediabetes who are overweight or obese. The precise mechanism(s) responsible for these metabolic effects and the potential metabolic benefits of NMN supplementation in other patient populations remain to be explored.

\section{REFERENCES AND NOTES}

1. J. Yoshino, K. F. Mills, M. J. Yoon, S. Imai, Nicotinamide mononucleotide, a key NAD+ intermediate, treats the pathophysiology of diet- and age-induced diabetes in mice. Cell Metab. 14, 528-536 (2011). doi:10.1016/j.cmet.2011.08.014 Medline

2. K. M. Ramsey, K. F. Mills, A. Satoh, S. Imai, Age-associated loss of Sirt1-mediated enhancement of glucose-stimulated insulin secretion in beta cell-specific Sirt1overexpressing (BESTO) mice. Aging Cell 7, 78-88 (2008). doi:10.1111/j.14749726.2007.00355.x Medline

3. A. P. Gomes, N. L. Price, A. J. Y. Ling, J. J. Moslehi, M. K. Montgomery, L. Rajman, J. P. White, J. S. Teodoro, C. D. Wrann, B. P. Hubbard, E. M. Mercken, C. M. Palmeira, R. de Cabo, A. P. Rolo, N. Turner, E. L. Bell, D. A. Sinclair, Declining NAD+ induces a pseudohypoxic state disrupting nuclear-mitochondrial communication during aging. Cell 155, 1624-1638 (2013). doi:10.1016/j.cell.2013.11.037 Medline

4. K. L. Stromsdorfer, S. Yamaguchi, M. J. Yoon, A. C. Moseley, M. P. Franczyk, S. C. Kelly, N. Qi, S. Imai, J. Yoshino, NAMPT-mediated NAD+ biosynthesis in adipocytes regulates adipose tissue function and multi-organ insulin sensitivity in mice. Cell Rep. 16, 1851-1860 (2016). doi:10.1016/j.celrep.2016.07.027 Medline

5. J. Yoshino, J. A. Baur, S. I. Imai, NAD+ intermediates: The biology and therapeutic potential of NMN and NR. Cell Metab. 27, 513-528 (2018). doi:10.1016/i.cmet.2017.11.002 Medline

6. S. Imai, J. Yoshino, The importance of NAMPT/NAD/SIRT1 in the systemic regulation of metabolism and ageing. Diabetes Obes. Metab. 15, 26-33 (2013). doi:10.1111/dom.12171 Medline

7. E. Verdin, NAD+ in aging, metabolism, and neurodegeneration. Science 350, 12081213 (2015). doi:10.1126/science.aac4854 Medline

8. E. Katsyuba, M. Romani, D. Hofer, J. Auwerx, NAD ${ }^{+}$homeostasis in health and disease. Nat Metab 2, 9-31 (2020). doi:10.1038/s42255-019-0161-5 Medline

9. M. R. McReynolds, K. Chellappa, J. A. Baur, Age-related NAD+ decline. Exp. Gerontol. 134, 110888 (2020). doi:10.1016/j.exger.2020.110888 Medline

10. G. M. Uddin, N. A. Youngson, D. A. Sinclair, M. J. Morris, Head to head comparison of short-term treatment with the $\mathrm{NAD}^{+}$precursor nicotinamide mononucleotide (NMN) and 6 weeks of exercise in obese female mice. Front. Pharmacol. 7, 258 (2016). doi:10.3389/fphar.2016.00258 Medline

11. K. F. Mills, S. Yoshida, L. R. Stein, A. Grozio, S. Kubota, Y. Sasaki, P. Redpath, M. E. Migaud, R. S. Apte, K. Uchida, J. Yoshino, S. I. Imai, Long-term administration of nicotinamide mononucleotide mitigates age-associated physiological decline in mice. Cell Metab. 24, 795-806 (2016). doj:10.1016/j.cmet.2016.09.013 Medline

12. American Diabetes Association, 2. Classification and Diagnosis of Diabetes: Standards of Medical Care in Diabetes-2020. Diabetes Care 43, S14-S31 (2020). doj:10.2337/dc20-S002 Medline

13. J. Irie, E. Inagaki, M. Fujita, H. Nakaya, M. Mitsuishi, S. Yamaguchi, K. Yamashita, 
S. Shigaki, T. Ono, H. Yukioka, H. Okano, Y. I. Nabeshima, S. I. Imai, M. Yasui, K. Tsubota, $\mathrm{H}$. Itoh, Effect of oral administration of nicotinamide mononucleotide on clinical parameters and nicotinamide metabolite levels in healthy Japanese men. Endocr. J. 67, 153-160 (2020). doi:10.1507/endocri.EJ19-0313 Medline

14. G. I. Smith, M. Shankaran, M. Yoshino, G. G. Schweitzer, M. Chondronikola, J. W. Beals, A. L. Okunade, B. W. Patterson, E. Nyangau, T. Field, C. B. Sirlin, S. Talukdar, M. K. Hellerstein, S. Klein, Insulin resistance drives hepatic de novo lipogenesis in nonalcoholic fatty liver disease. J. Clin. Invest. 130, 1453-1460 (2020). doi:10.1172/JCl134165 Medline

15. E. Fabbrini, F. Magkos, C. Conte, B. Mittendorfer, B. W. Patterson, A. L. Okunade, S. Klein, Validation of a novel index to assess insulin resistance of adipose tissue lipolytic activity in obese subjects. J. Lipid Res. 53, 321-324 (2012). doi:10.1194/ilr.D020321 Medline

16. A. Pannérec, L. Formicola, V. Besson, G. Marazzi, D. A. Sassoon, Defining skeletal muscle resident progenitors and their cell fate potentials. Development 140 , 2879-2891 (2013). doi:10.1242/dev.089326 Medline

17. M. Crisan, S. Yap, L. Casteilla, C.-W. Chen, M. Corselli, T. S. Park, G. Andriolo, B. Sun, B. Zheng, L. Zhang, C. Norotte, P.-N. Teng, J. Traas, R. Schugar, B. M. Deasy, S. Badylak, H.-J. Bühring, J.-P. Giacobino, L. Lazzari, J. Huard, B. Péault, A perivascular origin for mesenchymal stem cells in multiple human organs. Cell Stem Cell 3, 301-313 (2008). doi:10.1016/i.stem.2008.07.003 Medline

18. D. W. Frederick, E. Loro, L. Liu, A. Davila Jr., K. Chellappa, I. M. Silverman, W. J. Quinn III, S. J. Gosai, E. D. Tichy, J. G. Davis, F. Mourkioti, B. D. Gregory, R. W. Dellinger, P. Redpath, M. E. Migaud, E. Nakamaru-Ogiso, J. D. Rabinowitz, T. S. Khurana, J. A. Baur, Loss of NAD homeostasis leads to progressive and reversible degeneration of skeletal muscle. Cell Metab. 24, 269-282 (2016). doi:10.1016/i.cmet.2016.07.005 Medline

19. F. Magkos, G. Fraterrigo, J. Yoshino, C. Luecking, K. Kirbach, S. C. Kelly, L. de Las Fuentes, S. He, A. L. Okunade, B. W. Patterson, S. Klein, Effects of moderate and subsequent progressive weight loss on metabolic function and adipose tissue biology in humans with obesity. Cell Metab. 23, 591-601 (2016). doi:10.1016/i.cmet.2016.02.005 Medline

20. J. J. Nolan, B. Ludvik, P. Beerdsen, M. Joyce, J. Olefsky, Improvement in glucose tolerance and insulin resistance in obese subjects treated with troglitazone. $N$. Engl. J. Med. 331, 1188-1193 (1994). doi:10.1056/NEJM199411033311803 Medline

21. M. Razmara, C. H. Heldin, J. Lennartsson, Platelet-derived growth factor-induced Akt phosphorylation requires mTOR/Rictor and phospholipase C- $\gamma 1$, whereas S6 phosphorylation depends on mTOR/Raptor and phospholipase D. Cell Commun. Signal. 11, 3 (2013). doi:10.1186/1478-811X-11-3 Medline

22. B. H. Rauch, A. Weber, M. Braun, N. Zimmermann, K. Schrör, PDGF-induced Akt phosphorylation does not activate NF- $\mathrm{KB}$ in human vascular smooth muscle cells and fibroblasts. FEBS Lett. 481, 3-7 (2000). doi:10.1016/S0014-5793(00)019578 Medline

23. K. L. Hoehn, C. Hohnen-Behrens, A. Cederberg, L. E. Wu, N. Turner, T. Yuasa, Y. Ebina, D. E. James, IRS1-independent defects define major nodes of insulin resistance. Cell Metab. 7, 421-433 (2008). doi:10.1016/i.cmet.2008.04.005 Medline

24. C. Cantó, R. H. Houtkooper, E. Pirinen, D. Y. Youn, M. H. Oosterveer, Y. Cen, P. J. Fernandez-Marcos, H. Yamamoto, P. A. Andreux, P. Cettour-Rose, K. Gademann, C. Rinsch, K. Schoonjans, A. A. Sauve, J. Auwerx, The NAD precursor nicotinamide riboside enhances oxidative metabolism and protects against highfat diet-induced obesity. Cell Metab. 15, 838-847 (2012). doi:10.1016/i.cmet.2012.04.022 Medline

25. O. L. Dollerup, B. Christensen, M. Svart, M. S. Schmidt, K. Sulek, S. Ringgaard, H. Stødkilde-Jørgensen, N. Møller, C. Brenner, J. T. Treebak, N. Jessen, A randomized placebo-controlled clinical trial of nicotinamide riboside in obese men: Safety, insulin-sensitivity, and lipid-mobilizing effects. Am. J. Clin. Nutr. 108, 343-353 (2018). doi:10.1093/ajcn/nqy132 Medline

26. Y. S. Elhassan, K. Kluckova, R. S. Fletcher, M. S. Schmidt, A. Garten, C. L. Doig, D. M. Cartwright, L. Oakey, C. V. Burley, N. Jenkinson, M. Wilson, S. J. E. Lucas, I. Akerman, A. Seabright, Y.-C. Lai, D. A. Tennant, P. Nightingale, G. A. Wallis, K. N. Manolopoulos, C. Brenner, A. Philp, G. G. Lavery, Nicotinamide riboside augments the aged human skeletal muscle NAD+ metabolome and induces transcriptomic and anti-inflammatory signatures. Cell Rep. 28, 1717-1728.e6 (2019). doi:10.1016/i.celrep.2019.07.043 Medline

27. C. M. E. Remie, K. H. M. Roumans, M. P. B. Moonen, N. J. Connell, B. Havekes, J. Mevenkamp, L. Lindeboom, V. H. W. de Wit, T. van de Weijer, S. A. B. M. Aarts, E. Lutgens, B. V. Schomakers, H. L. Elfrink, R. Zapata-Pérez, R. H. Houtkooper, J. Auwerx, J. Hoeks, V. B. Schrauwen-Hinderling, E. Phielix, P. Schrauwen, Nicotinamide riboside supplementation alters body composition and skeletal muscle acetylcarnitine concentrations in healthy obese humans. Am. J. Clin. Nutr. 112, 413-426 (2020). doi:10.1093/ajcn/nqaa072 Medline

28. G. I. Smith, D. C. Polidori, M. Yoshino, M. L. Kearney, B. W. Patterson, B. Mittendorfer, S. Klein, Influence of adiposity, insulin resistance, and intrahepatic triglyceride content on insulin kinetics. J. Clin. Invest. 130, 3305-3314 (2020). doi:10.1172/JC1136756 Medline

29. J. Yoshino, S. Imai, Accurate measurement of nicotinamide adenine dinucleotide $\left(\mathrm{NAD}^{+}\right)$with high-performance liquid chromatography. Methods Mol. Biol. 1077, 203-215 (2013). doi:10.1007/978-1-62703-637-5 14 Medline

30. F. J. Naser, N. G. Mahieu, L. Wang, J. L. Spalding, S. L. Johnson, G. J. Patti, Two complementary reversed-phase separations for comprehensive coverage of the semipolar and nonpolar metabolome. Anal. Bioanal. Chem. 410, 1287-1297 (2018). doi:10.1007/s00216-017-0768-x Medline

31. S. J. Gelman, F. Naser, N. G. Mahieu, L. D. McKenzie, G. P. Dunn, M. G. Chheda, G. J. Patti, Consumption of NADPH for 2-HG synthesis increases pentose phosphate pathway flux and sensitizes cells to oxidative stress. Cell Rep. 22, 512-522 (2018). doi:10.1016/i.celrep.2017.12.050 Medline

32. S. Yamaguchi, M. P. Franczyk, M. Chondronikola, N. Qi, S. C. Gunawardana, K. L. Stromsdorfer, L. C. Porter, D. F. Wozniak, Y. Sasaki, N. Rensing, M. Wong, D. W. Piston, S. Klein, J. Yoshino, Adipose tissue NAD+ biosynthesis is required for regulating adaptive thermogenesis and whole-body energy homeostasis in mice. Proc. Natl. Acad. Sci. U.S.A. 116, 23822-23828 (2019). doi:10.1073/pnas.1909917116 Medline

33. C. Conte, E. Fabbrini, M. Kars, B. Mittendorfer, B. W. Patterson, S. Klein, Multiorgan insulin sensitivity in lean and obese subjects. Diabetes Care 35, 1316-1321 (2012). doi:10.2337/dc11-1951 Medline

34. S. Klein, L. Fontana, V. L. Young, A. R. Coggan, C. Kilo, B. W. Patterson, B. S. Mohammed, Absence of an effect of liposuction on insulin action and risk factors for coronary heart disease. N. Engl. J. Med. 350, 2549-2557 (2004). doi:10.1056/NEJMoa033179 Medline

\section{ACKNOWLEDGMENTS}

The authors thank Jennifer Shew, Freida Custodio, Drs. Adewole Okunade, Sean Johnson and Kyohei Tokizane, and Hanuye Cecilia Lei for their laboratory technical assistance, Sally Torbitzky, Zackary Boger, Miranda Giuffrida, Jessica Britt, and the Clinical and Translational Research Unit staff for their assistance in conducting the studies, the study subjects for their participation and Oriental Yeast Co., Ltd. (Tokyo, Japan) for providing placebo and NMN capsules. Funding: This study was supported by National Institutes of Health grants DK56341, DK104995, AG037457, AG047902, the UC San Diego IGM Genomics Center utilizing an Illumina NovaSeq 6000 that was purchased with funding from a National Institutes of Health SIG grant (\#S10 OD026929), and support from the Tanaka Fund and the Foundation for Barnes-Jewish Hospital. Author contributions: M.Y. and B.D.K. conducted the clinical studies. J.Y., M.P.F., K.F.M, B.W.P., G.P., M.S and T.P. performed the sample analyses. M.Y., J.Y., G.P., B.W.P., S.I., and S.K. analyzed and interpreted the data. M.Y., J.Y., S.I., and S.K. wrote the manuscript. S.K. designed and supervised the studies and obtained funding for the work. S.K. is the guarantor of this work, and as such, had full access to all the data in the study and takes responsibility for the integrity of the data and the accuracy of the data analysis. All authors critically reviewed and edited the manuscript. Competing interests: S.I. receives a part of patentlicensing fees from MetroBiotech (USA) and Teijin Limited (Japan) through Washington University. S.I. also serves as Invited Chief Scientist at the Institute for Biomedical Research and Innovation in Kobe, Japan, which does not involve competing interests. J.Y. is an inventor of a patent application related to NMN (\#20180228824). S.K. receives research support from Janssen Pharmaceuticals and serves on a scientific advisory board for Merck Sharp \& Dohme Corp. The other authors have nothing to disclose. Data and materials availability: All data in this paper are presented in the main text and supplementary materials. All 
figures are provided with individual values to have direct access to the raw data. This study was registered in ClinicalTrials.gov (https://clinicaltrials.gov/),

NCT03151239. The skeletal muscle sequencing datasets generated during the current study are available in the Gene Expression Omnibus (GEO) database (https://www.ncbi.nlm.nih.gov/geo) under accession no. GSE157988.

\section{SUPPLEMENTARY MATERIALS}

science.sciencemag.org/cgi/content/full/science.abe9985/DC1

Materials and Methods

Figs. S1 to S6

Tables S1 to S4

References (28-34)

MDAR Reproducibility Checklist

27 September 2020; accepted 8 April 2021

Published online 22 April 2021

10.1126/science.abe9985 
A

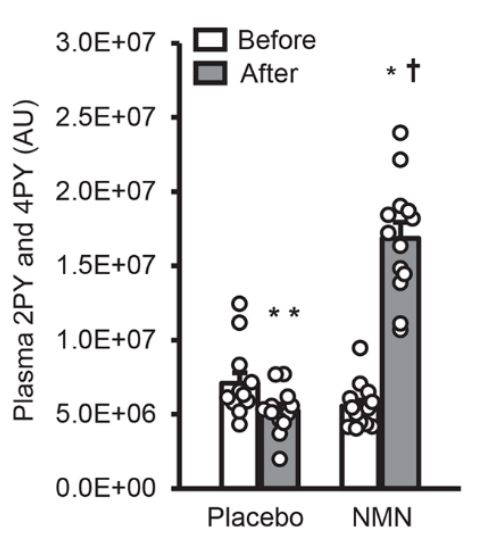

D

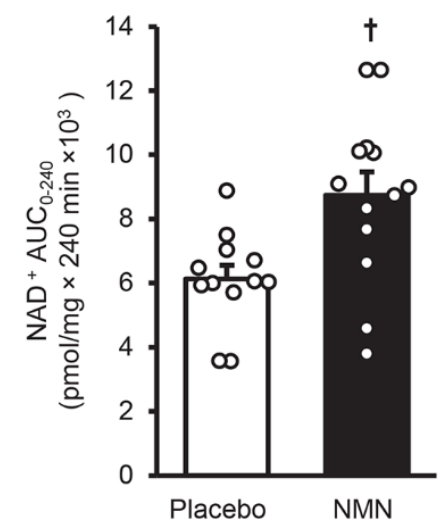

G

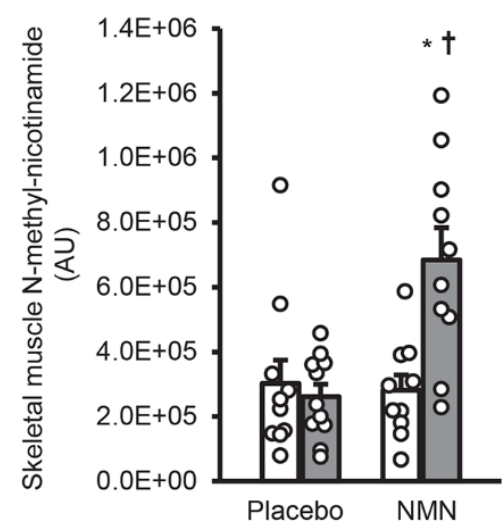

B

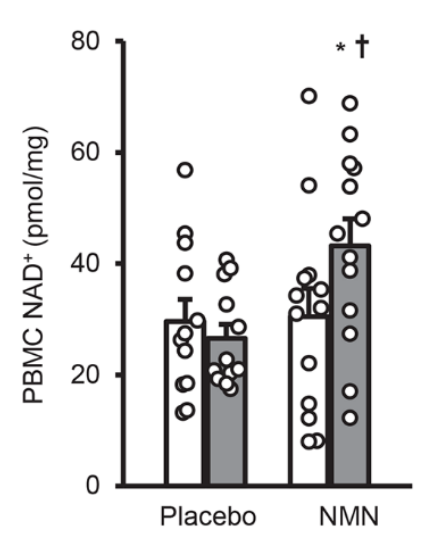

E

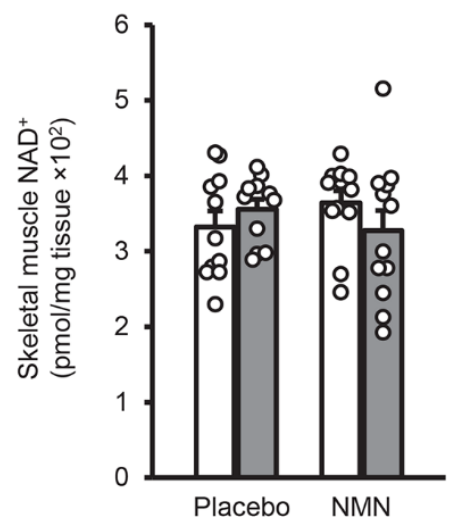

H

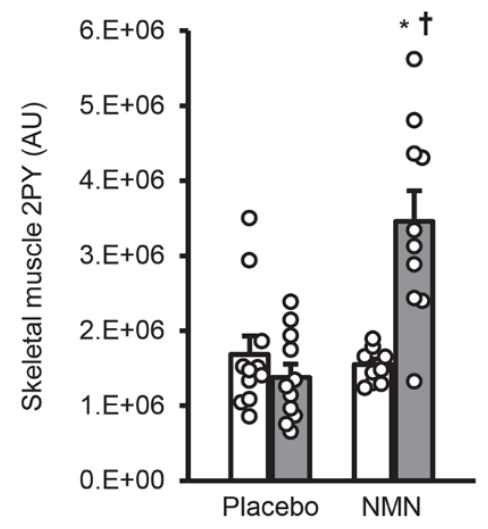

C

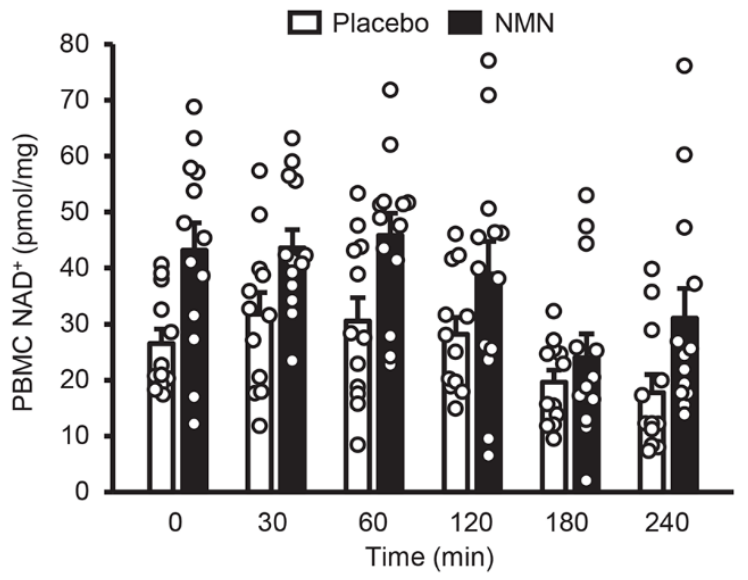

F

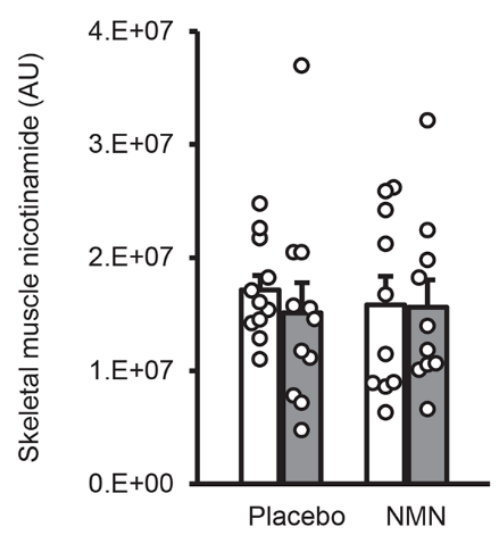

I

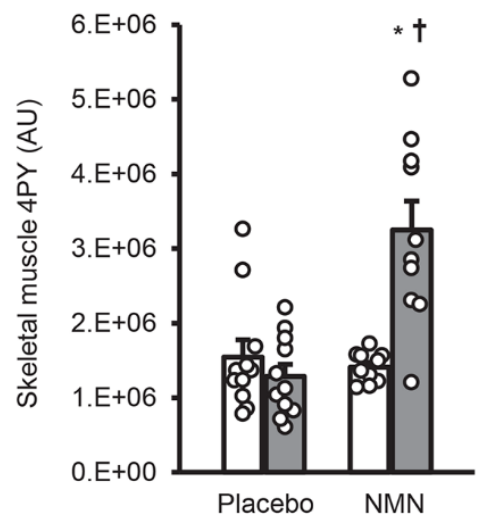

NMN metabolites and NAD ${ }^{+}$in plasma, PBMCs, and skeletal muscle. 
Fig. 1. NMN metabolites and NAD ${ }^{+}$in plasma, PBMCs, and skeletal muscle. (A) Plasma N-methyl2-pyridone-5-carboxamide (2PY) and $\mathrm{N}$-methyl-4-pyridone-5-carboxamide (4PY) before (white bars) and after (gray bars) treatment. (B) Basal peripheral blood mononuclear cell (PBMC) NAD ${ }^{+}$ content before (white bars) and after (gray bars) treatment. (C) PBMC NAD ${ }^{+}$content before (Time 0) and for 240 min after ingesting a placebo capsule (white bars) or NMN (250 mg) (black bars) at the end of 10 weeks of treatment with placebo or NMN. (D) PBMC NAD ${ }^{+}$area under the curve (AUC) for 240 min after ingesting a placebo capsule or $250 \mathrm{mg}$ of NMN. (E to I) skeletal muscle NAD', nicotinamide, N-methyl-nicotinamide, 2PY, and 4PY contents before (white bars) and after placebo or NMN treatment (gray bars). Two-way mixed model analysis of variance (ANOVA) with time (before- versus after- treatment) and group (placebo versus NMN) as factors was used to compare the effect of treatment with NMN and placebo on basal PBMC and tissue NAD ${ }^{+}$. A significant time by group interaction is followed by Tukey's post-hoc test to locate significant mean differences. A t test for independent samples (two-tailed) was used to determine differences between the mean PBMC AUC values in the two groups. *Value significantly different from corresponding before treatment value, $P<0.01 .{ }^{*}$ Value significantly different from corresponding before treatment value, $P<0.05$. tValue significantly different from corresponding value in the placebo group, $P<0.01$. Circles represent individual participant values: skeletal muscle NAD ${ }^{+}$content was measured in 11 placebo and 12 NMN participants; skeletal muscle NMN metabolites were measured in 11 placebo and 10 NMN participants; all other measurements are in 12 placebo and 13 NMN participants. Bars represent means \pm SEM. 
A

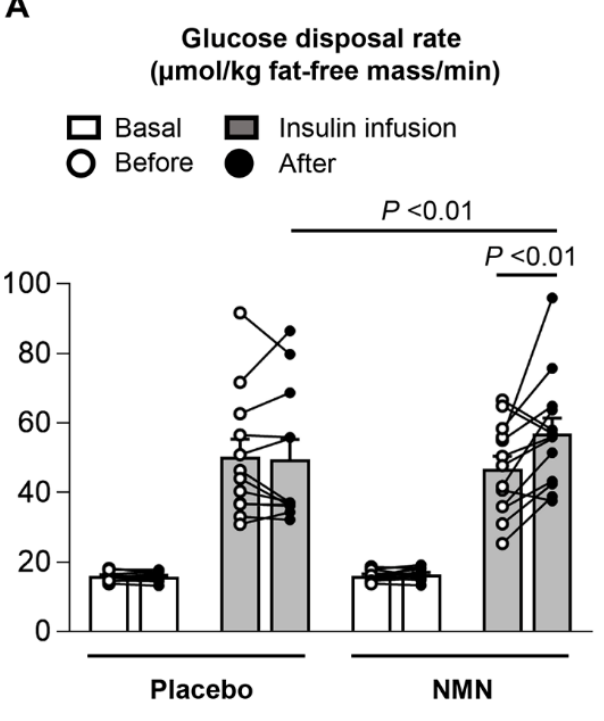

B
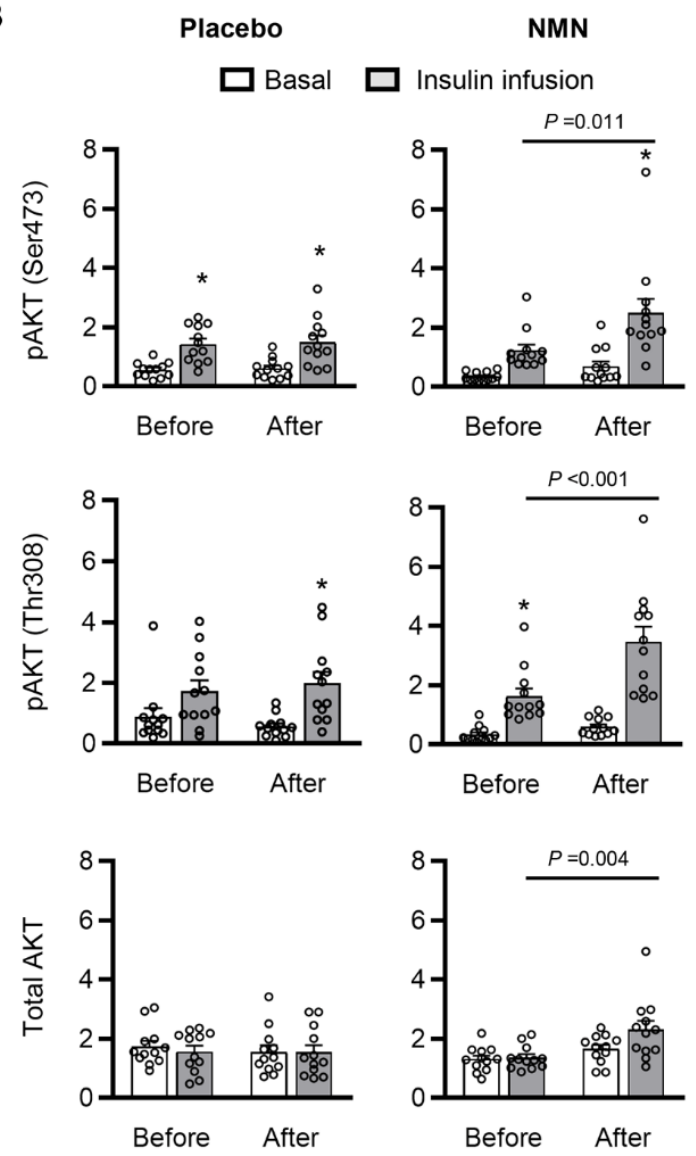

C

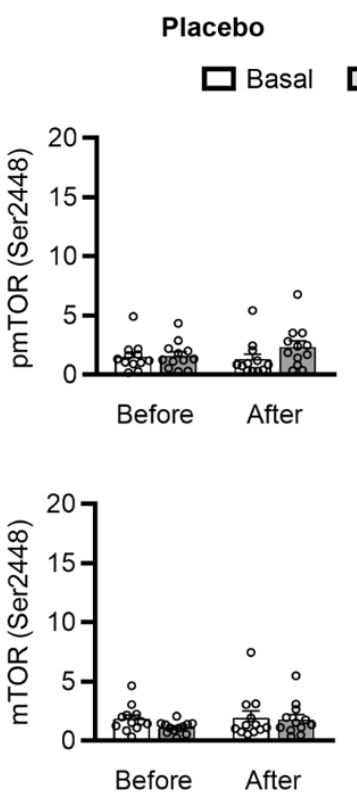

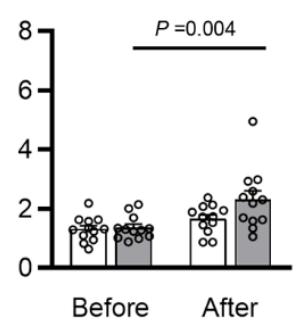

NMN

Insulin infusion
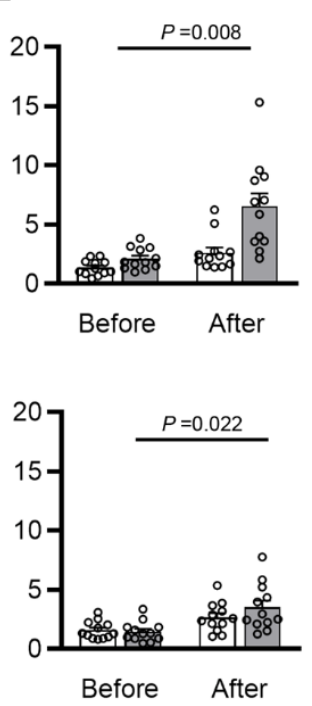

Insulin infusion
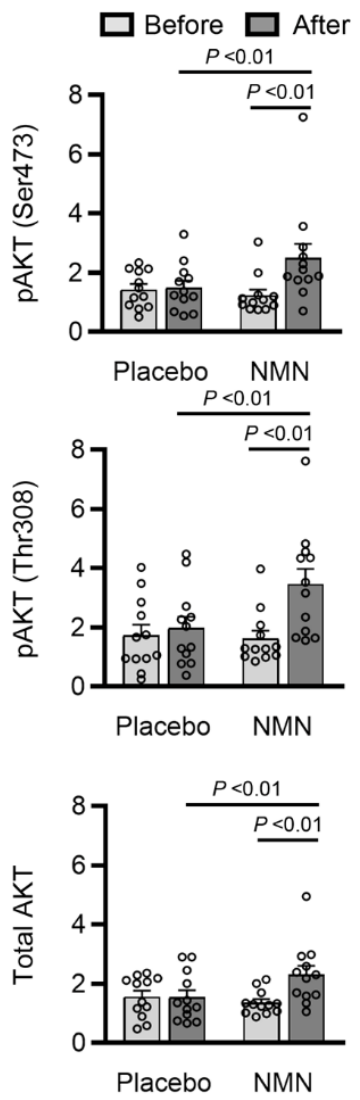

Insulin infusion
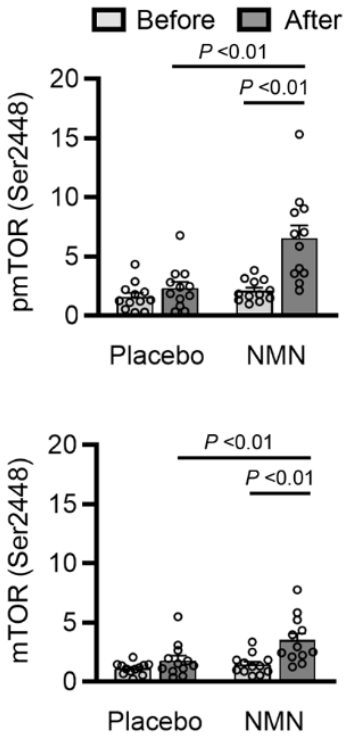

Effect pf NMN on skeletal muscle insulin sensitivity and signaling. 
Fig. 2. Effect of NMN on skeletal muscle insulin sensitivity and signaling. (A) Muscle insulin sensitivity, assessed as glucose disposal rate during basal conditions (white bar) and insulin infusion (gray bar) of a hyperinsulinemic-euglycemic clamp procedure. A three-way mixed model ANOVA with time (before- vs after- treatment), condition (basal vs insulin infusion), and group (placebo vs NMN) as factors, was used to compare the effect of NMN $(n=13)$ and placebo $(n=12)$ treatment on insulin-stimulated glucose disposal rate. A significant three-way interaction $(P=0.022)$ was followed by Tukey's post-hoc test to locate significant mean differences. Significant differences in mean values are represented by a line above the bars with the corresponding $P$ value. (B) Western blot densitometric analyses of phosphorylated AKT serine-473 (pAKT Ser473), threonine-308 (pAKT Thr308) and mTOR serine-2448 (pmTOR Ser2448), and total AKT and mTOR relative to ACTIN in skeletal muscle during basal conditions and insulin infusion before and after 10 weeks of treatment with placebo $(n=12)$ or NMN $(n=12)$. Raw images for individual Western blot analysis are provided in the supplementary materials (fig. S3). One-way ANOVA was performed to determine differences in the quantity of phosphorylated AKTs among each combination of time and condition (during basal and insulin infusion before and after each treatment) within each group. Following a significant omnibus test, Tukey's post-hoc test was used to locate significant mean differences. (C) Western blot densitometric analyses of pAKT Ser473, pAKT Thr308, pmTOR Ser2448, and total AKT and mTOR relative to ACTIN in skeletal muscle during insulin infusion before (light gray bars) and after (dark gray bars) placebo $(n=12)$ or NMN $(n=12)$ treatment. Two-way mixed model ANOVA with time (before- vs. after- treatment) and group (placebo vs NMN) as factors was used to determine the significance of differences between variables. A significant two-way interaction was followed by Tukey's post-hoc test to locate significant mean differences. Significant differences in mean values are represented by a line above the bars with the corresponding $P$ value. *Value significantly different from the corresponding basal value, $P<0.05$. Circles represent individual participant values. Bars represent means \pm SEM. 
A

Placebo

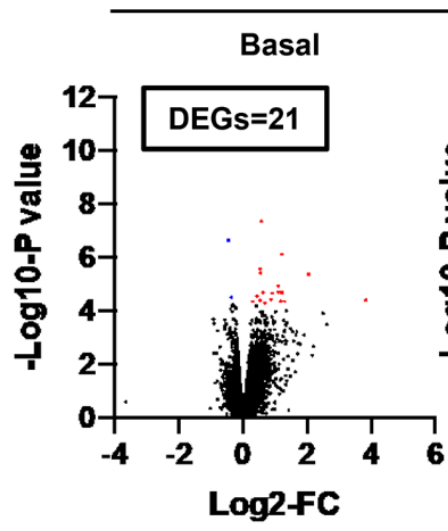

B

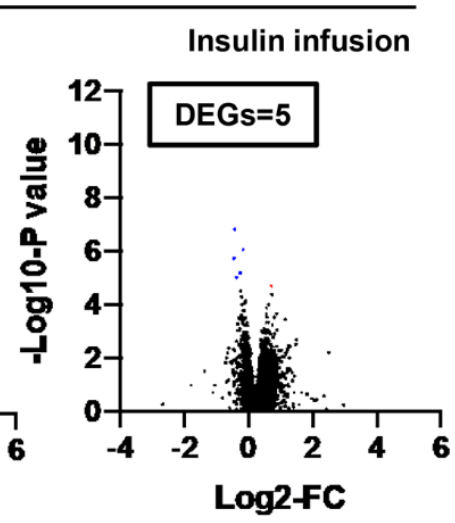

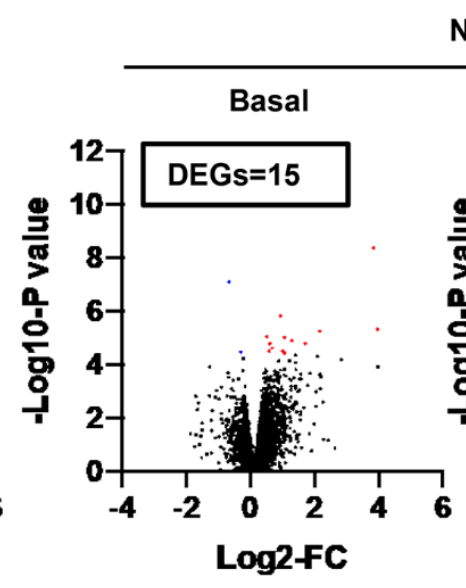

C
NMN

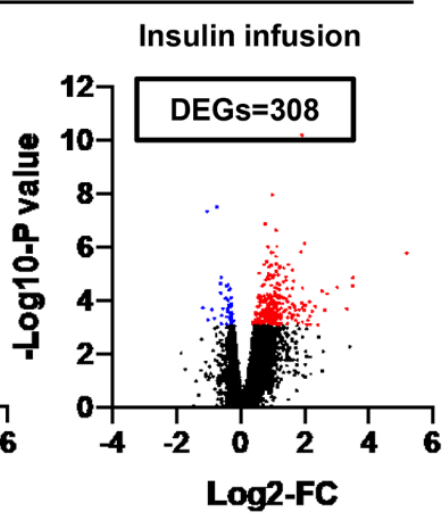

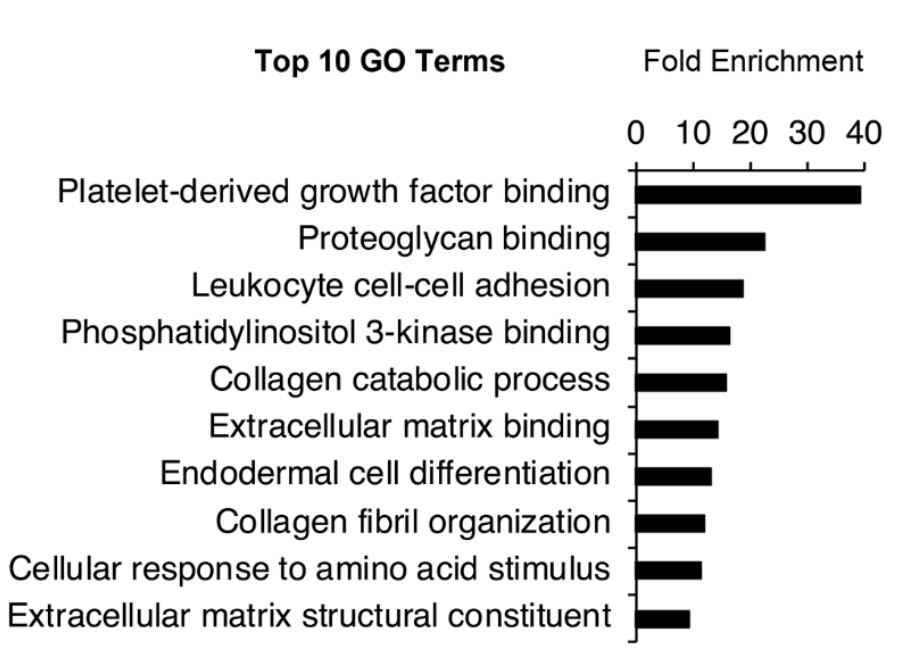

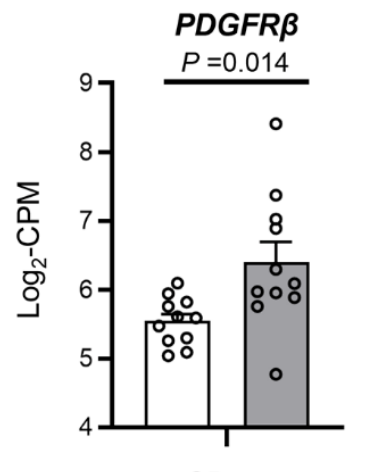

Before $\square$ After
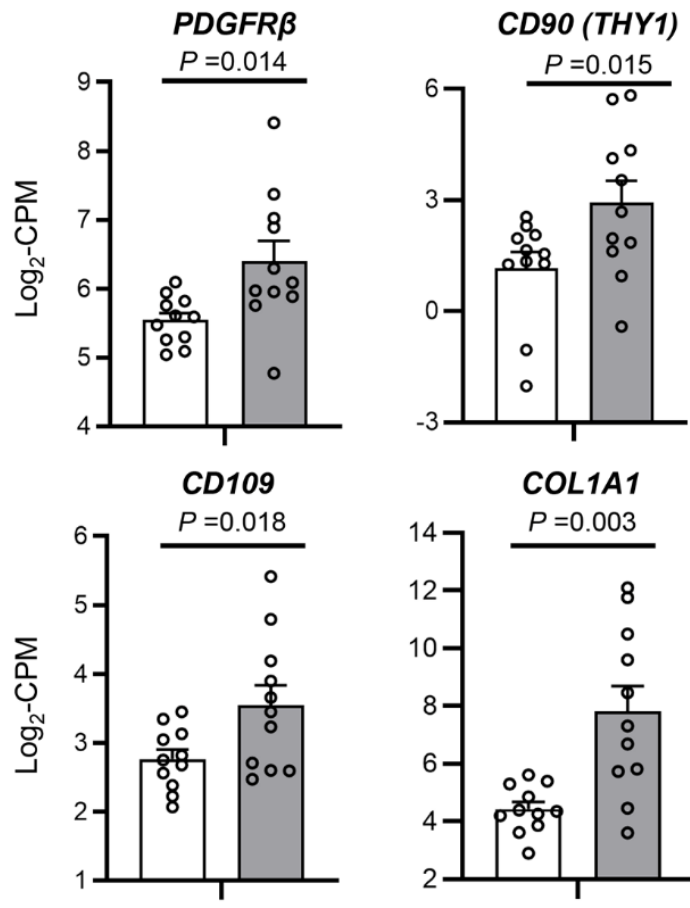

Effects of NMN supplementation on skeletal muscle global transcriptome profile.
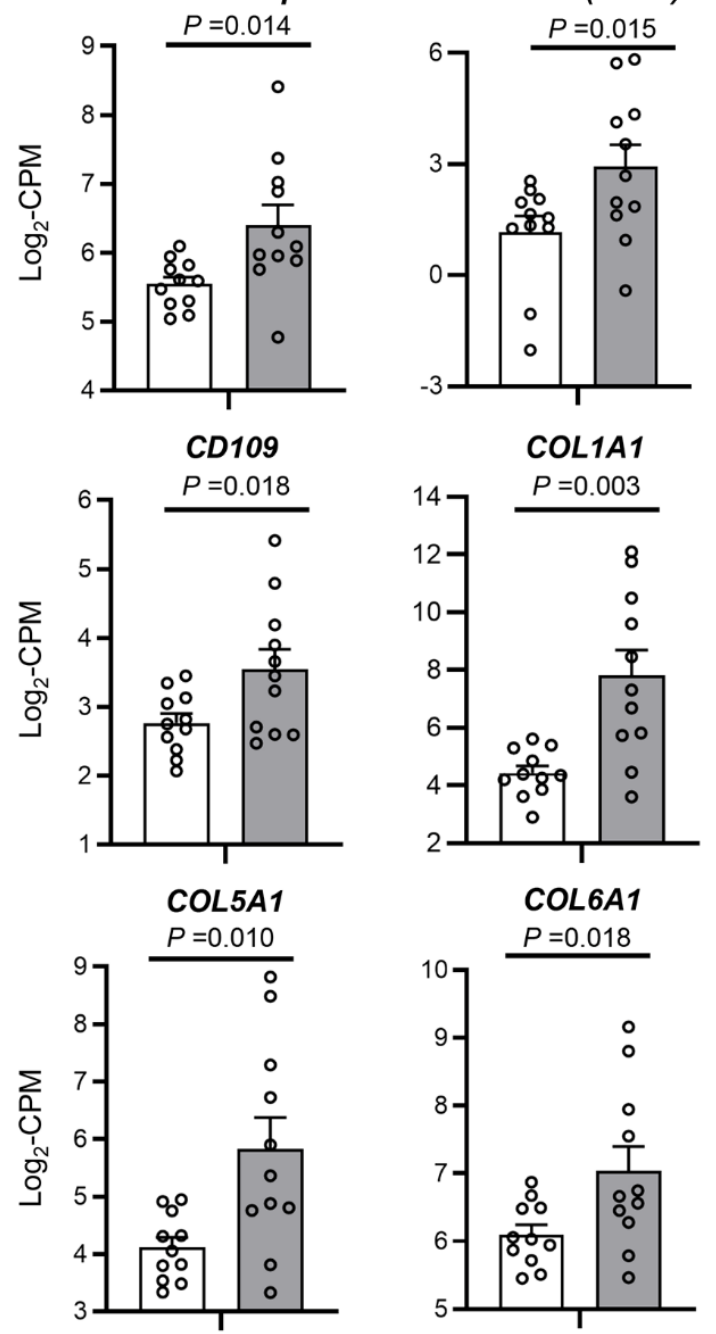
Fig. 3. Effects of NMN on skeletal muscle global transcriptome profile. Skeletal muscle tissue obtained during basal conditions and insulin infusion before and after treatment in placebo $(n=12)$ and NMN groups $(n=11)$ were evaluated by using RNA-sequencing (RNA-seq). (A) Volcano plots of RNA-seq data of skeletal muscle with $\log _{2}$-fold change (FC) (X-axis) and $-\log _{10}$ - $P$ value ( $Y$-axis). The number of differentially expressed genes (DEGs, FDR<0.05) between before and after treatment during basal conditions and during insulin infusion are shown in boxes in each panel. Significantly upregulated and down-regulated DEGs between before and after treatment in the placebo and NMN groups are shown as red and blue dots, respectively. (B) The top 10 Gene Ontology (GO) terms ranked by fold-enrichment. (C) Skeletal muscle gene expression of selective proteins involved in PDGF signaling and muscle remodeling during insulin infusion before and after NMN treatment in each participant. PDGFR $\beta, C D 90, C D 109, C O L 1 A 1, C O L 5 A 1$, and COL6A1 were identified as DEGs (table S2). Gene expression is presented as $\log _{2}$-transformed counts per million (CPM) reads. Lines represent values of individual participants before and after NMN treatment. A t test for paired samples (two-tailed) was performed to determine the difference between before and after NMN treatment for each gene; corresponding $P$ values are indicated above the black lines. 
Table 1. Body composition and basal metabolic variables. Values are means \pm SEM. Metabolic variables were measured before and after placebo $(n=12)$ or NMN $(n=13)$ treatment. A two-way mixed model ANOVA was used to evaluate the effect of NMN supplementation on each outcome. The effect of NMN on these variables was not different from placebo. Abbreviation: FFM, fat-free mass.

\begin{tabular}{|c|c|c|c|c|c|c|c|}
\hline & \multicolumn{2}{|c|}{ Placebo } & \multicolumn{2}{|c|}{ NMN } & \multicolumn{3}{|c|}{ RM-ANOVA } \\
\hline & Before & After & Before & After & $P_{\text {group }}$ & $\mathrm{P}_{\text {time }}$ & $\mathrm{P}_{\text {group x time }}$ \\
\hline Age (yrs) & $61 \pm 5$ & - & $62 \pm 4$ & - & - & - & - \\
\hline Body weight (kg) & $87 \pm 3$ & $87 \pm 3$ & $89 \pm 4$ & $89 \pm 4$ & 0.764 & 0.917 & 0.941 \\
\hline Body mass index $\left(\mathrm{kg} / \mathrm{m}^{2}\right)$ & $33.4 \pm 1.0$ & $33.3 \pm 0.9$ & $33.7 \pm 1.4$ & $33.8 \pm 1.5$ & 0.928 & 0.917 & 0.941 \\
\hline Fat mass (kg) & $40.3 \pm 2.0$ & $39.9 \pm 2.0$ & $42.8 \pm 2.9$ & $42.9 \pm 3.0$ & 0.547 & 0.672 & 0.702 \\
\hline Fat free mass (kg) & $45.6 \pm 0.9$ & $46.1 \pm 0.7$ & $44.8 \pm 1.2$ & $45.5 \pm 1.3$ & 0.654 & 0.011 & 0.846 \\
\hline Intrahepatic triglyceride content (\%) & $14.8 \pm 2.0$ & $15.7 \pm 2.5$ & $6.3 \pm 1.2$ & $6.8 \pm 1.3$ & 0.003 & 0.385 & 0.461 \\
\hline $\begin{array}{l}\text { Intra-abdominal adipose tissue volume } \\
\left(\mathrm{cm}^{3}\right)\end{array}$ & $1,576 \pm 71$ & $1,669 \pm 95$ & $1,492 \pm 213$ & $1,504 \pm 214$ & 0.265 & 0.180 & 0.186 \\
\hline Systolic blood pressure $(\mathrm{mmHg})$ & $128 \pm 4$ & $131 \pm 4$ & $126 \pm 5$ & $125 \pm 5$ & 0.089 & 0.813 & 0.686 \\
\hline Diastolic blood pressure $(\mathrm{mmHg})$ & $73 \pm 3$ & $77 \pm 3$ & $73 \pm 3$ & $75 \pm 3$ & 0.832 & 0.286 & 0.701 \\
\hline $\mathrm{HbA1C}(\%)$ & $5.5 \pm 0.1$ & $5.5 \pm 0.1$ & $5.7 \pm 0.1$ & $5.5 \pm 0.1$ & 0.407 & 0.008 & 0.396 \\
\hline Glucose $(\mathrm{mmol} / \mathrm{L})$ & $5.7 \pm 0.2$ & $5.6 \pm 0.2$ & $5.7 \pm 0.1$ & $5.6 \pm 0.2$ & 0.906 & 0.379 & 0.836 \\
\hline Insulin $(\mu \mathrm{U} / \mathrm{mL})$ & $16.7 \pm 2.0$ & $17.2 \pm 2.5$ & $13.6 \pm 1.9$ & $15.8 \pm 2.7$ & 0.400 & 0.122 & 0.335 \\
\hline Free fatty acids $(\mathrm{g} / \mathrm{L})$ & $0.198 \pm 0.018$ & $0.183 \pm 0.011$ & $0.192 \pm 0.014$ & $0.193 \pm 0.015$ & 0.973 & 0.493 & 0.492 \\
\hline Triglyceride (mmol/L) & $1.63 \pm 0.22$ & $1.48 \pm 0.23$ & $1.39 \pm 0.25$ & $1.26 \pm 0.18$ & 0.289 & 0.107 & 0.554 \\
\hline HDL-cholesterol (mmol/L) & $1.28 \pm 0.07$ & $1.34 \pm 0.07$ & $1.25 \pm 0.10$ & $1.30 \pm 0.10$ & 0.804 & 0.071 & 0.930 \\
\hline High molecular weight adiponectin $(\mathrm{Hg} / \mathrm{mL})$ & $4.02 \pm 0.53$ & $3.93 \pm 0.51$ & $3.90 \pm 0.49$ & $3.86 \pm 0.52$ & 0.900 & 0.564 & 0.843 \\
\hline Leptin $(n g / m L)$ & $83.5 \pm 8.1$ & $82.4 \pm 6.8$ & $88.1 \pm 6.7$ & $88.5 \pm 7.3$ & 0.589 & 0.916 & 0.811 \\
\hline Basal glucose Ra ( $\mu \mathrm{mol} / \mathrm{kg} F F M / m i n)$ & $15.5 \pm 0.4$ & $15.4 \pm 0.4$ & $15.7 \pm 0.4$ & $16.1 \pm 0.5$ & 0.469 & 0.459 & 0.311 \\
\hline Basal palmitate Ra ( $\mu \mathrm{mol} / \mathrm{kg} \mathrm{FFM} / \mathrm{min})$ & $2.66 \pm 0.29$ & $2.23 \pm 0.17$ & $2.28 \pm 0.21$ & $2.21 \pm 0.18$ & 0.455 & 0.098 & 0.247 \\
\hline
\end{tabular}

